\title{
On Continuation of Regular Solutions of Linear Partial Differential Equations
}

by

\author{
Akira KANEKO*
}

Here we briefly introduce the speaker's recent works on continuation of regular solutions of linear partial differential equations with real analytic coefficients. The method of argument is deeply concerned with the non-characteristic boundary value problem for hyperfunction solutions. First we intuitively compare this new method with the old one which owes much to Grušin [2] and was employed in the case of constant coefficients. Then we give results on hyperfunction boundary value problem as our main tool. Finally we give the main results and prospects on continuation of real analytic solutions.

1. For comparison we cite one typical result from the case of constant coefficients. Others may be found in [2], [4], [6], [7], [8].

Theroem A ([4]). Let $p(D)$ be a single linear partial differential operator with constant coefficients. Then the following are equivalent.

1) Every real analytic solution of $p(D) u=0$ defined outside a compact convex set $K$ is continued analytically to $K$.

2) $p$ has no elliptic factor.

Let us shortly review the method of proof of the main part 2) $\Rightarrow 1$ ) of this theorem. For the sake of simplicity we assume that $p$ is irreducible. Let $u$ be a real analytic solution of $p(D) u=0$ defined on $U \backslash K$, where $U$ is a neighborhood of $K$. We can extend $u$ to $U$ as a hyperfunction. Let $[u]$ be one of such extension. Then $p(D)[u]$ is a hyper-

Received June 25, 1976.

* Deparment of Mathematics, College of General Education, University of Tokyo. 
function with support in $K$ and is determined only modulo $p(D) \mathscr{B}[K]$. Here we let $\mathscr{B}[K]$ denote the hyperfunctions with support in $K$. To prove the theorem we must show that under the given condition on $p$ the set of $p(D)[u] \bmod p(D) \mathscr{B}[K]$ contains zero as a representative. To annihilate the ambiguity we apply the Fourier transform and restrict to the variety $p(\zeta)=0$. Then we obtain a uniquely defined holomorphic function $F(\zeta)$ on the variety. Translating the assumption on $p$ and the analyticity of $u$ outside $K$ to a kind of growth condition for $F(\zeta)$ and employing the Phragmén-Lindelöf type principle, we nullify this holomorphic function. Then the Fundamental Principle of Ehrenpreis-Palamo$d o v$ assures the existence of $[u]$ satisfying $p(D)[u]=0$. Finally a result on propagation of regularity guarantees the analyticity of $[u]$ even on $K$. (For further interesting interpretation see [5].)

If we attack the problem in the case of variable coefficients, we must translate each of these steps to a method applicable to such operators. Recent works of the speaker have suggested the following procedure when the singularity $K$ is contained in a noncharacteristic hyperplane: Let $x_{1}=0$ be the one containing $K$ and let $u$ be a real analytic solution of $p(x, D) u=0$ defined on $U \backslash K$. Then we can take a unique extension [u] of $u$ to $U$ characterized by the identity

$$
p(x, D)[u]=\sum_{j=0}^{m-1} \delta^{(j)}\left(x_{1}\right) u_{j}\left(x^{\prime}\right),
$$

where $m$ is the order of $p$ and $\left\{u_{j}\left(x^{\prime}\right)\right\}$ are the difference of the boundary values of $u$ to the hyperplane $x_{1}=0$ from both sides. Here the boundary value means that of Komatsu-Kawai [12]. Therefore we only have to examine whether $\left\{u_{j}\left(x^{\prime}\right)\right\}$ vanish or not, and thus we have bypassed the Fourier transform and the Fundamental Principle. Instead of the growth condition on the Fourier image, the assumption on $p$ and the analyticity of $u$ reflect on the analytic singular spectrum (analytic wave front set) of the boundary values $\left\{u_{j}\left(x^{\prime}\right)\right\}$. Then, instead of the Phragmén-Lindelöf type principle we can employ the Holmgren type theorem of Kashiwara-Kawai (see [13]) concerning the unique continuation property for analytic parameters. The above is the outline of the story in the case of variable coefficients.

Thus we examine in this paragraph the analytic singular spectrum 
of boundary values of solutions. Let $p(x, D)$ be a given operator of $m$-th order with real analytic coefficients. Assume that $S=\left\{x_{1}=0\right\}$ is non-characteristic with respect to $p$.

Definition B. Consider the set of points $\left(x, \sqrt{-1} \xi^{\prime} d x^{\prime} \infty\right) \in\left\{x_{1}\right.$ $>0\} \times \sqrt{-1} S_{\infty}^{* n-2}$ such that the characteristic equation $p_{m}\left(x, \zeta_{1}, \xi^{\prime}\right)=0$ for $\zeta_{1}$ has a root with positive (resp. non-negative) imaginary part. (Here $p_{m}$ denotes the principal part of $p$.) Take its closure in $\mathbb{R}^{n} \times \sqrt{-1} S_{\infty}^{* n-2}$ and restrict to $x_{1}=0$. We let $V_{S, A}^{+}(p)$ (resp. $\left.V_{S, B}^{+}(p)\right)$ denote the set of points $\left(x^{\prime}, \sqrt{-1} \xi^{\prime} d x^{\prime} \infty\right) \in \boldsymbol{R}^{n-1} \times \sqrt{-1} \mathbf{S}_{\infty}^{* n-2}$ obtained in this way.

Then we have ([10])

Theorem C. Let $u$ be a real analytic (resp. hyperfunction) solution of $p(x, D) u=0$ on $x_{1}>0$. Then the analytic singular spectrum of the boundary values of $u$ to $x_{1}=O$ is contained in $V_{S, A}^{+}(p)$ (resp. $\left.V_{S, B}^{+}(p)\right)$. (The analytic singular spectrum of boundary values of solutions on $x_{1}<0$ can be estimated in the same way by $V_{S, A}^{-}(p)$ (resp. $\left.V_{\vec{S}, B}(p)\right)$ which is defined exchanging the sign of the characteristic roots in the above definition.)

For example, consider the ultrahyperbolic operator $p(D)=D_{1}{ }^{2}+\cdots$ $+D_{k}{ }^{2}-D_{k+1}^{2}-\cdots-D_{n}{ }^{2}$. We have

$$
V_{S, A}^{ \pm}(p)=\left\{\left(x^{\prime}, \sqrt{-1} \xi^{\prime} d x^{\prime} \infty\right) ; \xi_{2}^{2}+\cdots+\xi_{k}^{2} \geqq \xi_{k+1}^{2}+\cdots+\xi_{n}^{2}\right\} .
$$

On the other hand, $V_{S, \bar{B}}(p)$ agrees with the whole cosphere bundle. This suggests the possibility of the mixed problem only for the nonanalytic boundary data. Next consider the generalized Lewy-Mizohata operator $p(x, D)=D_{1}+\sqrt{-1} x_{1}{ }^{2 k+1} D_{2}$. Then we have

$$
V_{S, B}^{ \pm}(p)=\left\{\left(x^{\prime}, \sqrt{-1} \xi^{\prime} d x^{\prime} \infty\right) ; \xi_{2} \geqq 0\right\}=V_{S, A}^{ \pm}(p) .
$$

From this estimate we conclude that we cannot find a hyperfunction solution $E$ of $p(x, D) E=\delta(x)$. In fact the difference of the boundary values of $E$ would then become a constant multiple of $\delta\left(x^{\prime}\right)$ contrary to the above estimate. 
The proof of Theorem $C$ is carried out employing Green's formula with the solution of the dual boundary value problem whose boundary data consist of the component of the curved wave decomposition of $\delta\left(x^{\prime}\right)$. Its solvability follows from the following theorem ([10]) extending the result of Bony-Schapira [1].

Definition $\mathbb{D}$. Let $I \subset \mathbb{R}^{n-1} \times \sqrt{-1} \mathbf{S}_{\infty}^{* n-2}$ be an open subset of the cosphere bundle of $\boldsymbol{R}^{n-1}$. We say that $p$ is I-semihyperbolic (resp. Isemitonnel) to the positive side of $x_{1}=0$ if for every compact subset $L \subset I$ there exists $\varepsilon_{L}>0$ such that all the roots of the characteristic equation $p_{m}\left(x, \zeta_{1}, \xi^{\prime}\right)=0$ for $\zeta_{1}$ have non-negative (resp. positive) imaginary parts when $\left(x^{\prime}, \sqrt{-1} \xi^{\prime} d x^{\prime} \infty\right) \in L$ and $0<x_{1} \leqq \varepsilon_{L}$.

Theorem E. Assume that $p$ is I-semihyperbolic (resp. I-semitonnel) to the positive side of $x_{1}=0$. Then for every boundary data $\left\{u_{j}\left(x^{\prime}\right)\right\}_{j=0}^{m-1}$ whose analytic singular spectrum is contained in $I$, we can find a unique local hyperfunction (resp. real analytic) solution of the boundary value problem

$$
\left\{\begin{array}{l}
p(x, D) u=0 \text { on } x_{1}>0, \\
\left.B_{j}(x, D) u\right|_{x_{1 \rightarrow+0}}=u_{j}\left(x^{\prime}\right), \quad j=0, \cdots, m-1,
\end{array}\right.
$$

where $\left\{B_{j}\right\}$ denotes a normal system of boundary operators with real analytic coefficients.

2. Employing the result and the reasoning of the preceding section we can give the following results ([10]).

$$
\begin{aligned}
\operatorname{Definition} \mathbb{F}_{0} \quad & V_{S, A}(p)=V_{S, A}^{+}(p) \cup V_{S, A}^{-}(p), \\
& V_{S, B}(p)=V_{S, B}^{+}(p) \cup V_{S, B}^{-}(p) .
\end{aligned}
$$

Theorem G. Let $K$ be a closed set in the non-characteristic hyperplane $S=\left\{x_{1}=0\right\}$. Assume that $K$ is contained in one side of an analytic hypersurface $\varphi\left(x^{\prime}\right)=0$ in $x_{1}=0$ passing through the origin, that is, $\varphi(0)=0$ and $d \varphi(0) \neq 0$. Assume that either of the points 
$(0, \pm \sqrt{-1} d \varphi(0) \infty)$ lies outside the set $V_{S, A}(p)$ (resp. $\left.V_{S, B}(p)\right)$. Then every real analytic (resp. hyperfunction) solution $u$ of $p(x, D) u=0$ defined on a neighborhood of the origin except on $K$ can be uniquely continued as a hyperfunction solution to $K$ in a smaller neighborhood.

In the extreme case $K=S=\left\{x_{1}=0\right\}$ we have

Theorem H. If $V_{S, A}^{+}(p) \subset\left[C V_{\overline{S, B}}^{-}(p)\right]^{a} \quad\left(\right.$ resp. $\quad V_{S, B}^{+}(p) \subset$ $\left.\left[C V_{\bar{S}, B}(p)\right]^{a}\right)$, then every real analytic (resp. hyperfunction) solution of $p(x, D) u=0$ on $x_{1}>0$ can be uniquely continued to $x_{1} \leqq 0$ as a hyperfunction solution. If we can take $V_{\vec{S}, A}(p)$ instead of $V_{\vec{S}, B}(p)$, then the continued solution is real analytic on $x_{1}<0$. (Here $C$ denotes the complement in $\boldsymbol{R}^{n-1} \times \sqrt{-1} \mathbf{S}_{\infty}^{* n-2}$ and a denotes the antipodal map.)

Let us consider a few examples. The first is $D_{1}+\sqrt{-1} x_{1}{ }^{2 k+1} D_{2}$ on $\boldsymbol{R}^{2}$. Theorem $H$ asserts that every solution of this operator on $x_{1}>0$ $\left(x_{1}<0\right)$ can be uniquely continued to $x_{1} \leqq 0 \quad\left(x_{1} \geqq 0\right)$. The second is the Tricomi operator $D_{1}{ }^{2}-x_{1} D_{2}{ }^{2}$. Since $V_{S, A}^{+}(p)=\phi$ for this operator, it follows that every real analytic solution on $x_{1}>0$ can be uniquely continued to $x_{1} \leqq 0$ (even real analytically). This latter case was independently discussed by P. Schapira by a different method (private communication).

In the case of Theorem $G$ separate consideration on propagation of analyticity is needed in order to assure the analyticity of the continued solution on $K$. As a special case we have the following theorem on removable isolated singularity ([9]).

Theorem I. Assume that the principal part $p_{m}(x, D)$ is of principal type and has real coefficients. Assume that there exists a direction $\xi^{\prime} \in \mathbb{R}^{n-1}$ such that the algebraic equation $p_{m}\left(x, \zeta_{1}, \xi^{\prime}\right)=0$ for $\zeta_{1}$ has real distinct roots on a neighborhood of the origin. Then every real analytic (infinitely differentiable) solution $u$ of $p(x, D) u=0$, defined on a neighborhood of the origin except the origin itself, can be continued to the origin as a real analytic (an infinitely differentiable) solution. 
In this case the propagation of regularity is readily shown by employing the fundamental solution. In the case of infinitely differentiable solutions we must make a suitable consideration of the Phragmén-Lindelöf type replacing the Holmgren type theorem with respect to analytic parameters. For, in this case the difference of the boundary values is only a hyperfunction with infinitely differentiable parameters in a very weak sense. The same technique allows us to show that classical solutions can be continued to the origin as distribution solutions. As for the continuation of infinitely differentiable solutions, Theorem I may be extended to more general operators and more general singularities by introducing a kind of Levi condition.

The results of this paragraph are almost best except one crucial point that the irreducibility of the operator is not discussed at all. Hence Theorem $\mathrm{A}$ is not covered. We hope that in future a theory of a kind of monodromy groups or Galois groups acting on the boundary data will overcome this difficulty.

3. The problem of continuation of solutions to a given set is rephrased conversely as the problem of determining whether the given set may contain a singularity of a solution of the equation. Thus the problem may be solved completely if we determine the whole singularity of the solutions of a given equation. In such an approach it is important to consider the most elementary singularity, or the singularity with minimal dimension. General singularity may be given by composing them by integration. For example, consider the single elliptic equation. It never appeared in our consideration. The reason is that it has a solution with an isolated singularity, that is, an analytic set of dimension equal to zero. Thus it has a solution with singularity of any given shape. As for the general operator $p(x, D)$, we conjecture that there exists a natural number $r(p)$ such that every singularity of real analytic solution of $p(x, D) u=0$ has dimension not less than $r(p)$ and that the singularity of dimension $r(p)$ becomes an analytic set.

For the singularity contained in a non-characteristic analytic hypersurface $S$, we can apply the result of $\mathbf{2}^{\circ}$ and support this conjecture. By a suitable real analytic coordinate transformation we can assume that 
$S=\left\{x_{1}=0\right\} . \quad$ First we give

Definition J. A submanifold $C$ of $S$ of class $C^{1}$ is called reakly timelike with respect to $p$ if its conormal elements are contained in $V_{S, A}(p)$. It is called timelike with respect to $p$ if its conormal elements are contained in

$$
V_{S, A}^{0}(p)=\left\{\left(x^{\prime}, \sqrt{-1} \xi^{\prime} d x^{\prime} \infty\right) \in V_{S, A}(p) ; p_{m}\left(0, x^{\prime}, \xi_{1}, \xi^{\prime}\right) \neq 0 \text { if } \xi_{1} \in \mathbb{R}\right\} .
$$

The latter definition agrees with the one given by John [1] in the general case

Definition K. We define $r_{S}(p)$ (resp. $r_{s}^{0}(p)$ ) to be the minimal value of the codimension in $\sqrt{-1} S_{\infty}^{* n-2}$ of concentric subsphere contained in the general fibre of $V_{S, A}(p)$ (resp. $\left.V_{S, A}^{0}(p)\right)$.

We have ([11])

Theorem L. Assume that there exists a real analytic solution $u$ of $p(x, D) u=0$ such that $C$ is a weak singularity (that is, $u$ can be extended as a distribution on a neighborhood of $C$ ) and unremovable as a hyperfunction solution. Then $C$ is weakly timelike and the dimension of $C$ is not less than $r_{S}(p)$. If $\operatorname{dim} C=r_{S}(p)$, then $C$ is an analytic submanifold.

The first part follows directly from Theorem G. The last part follows from Theorem $\mathrm{C}$ and the following

Lemma M. Let $v(t, x)$ be a distribution on a neighborhood of the origin of $\mathbb{R}^{n+1}$. Assume that supp $v$ is non-void and contained in a continuous hypersurface $C=\{t=\varphi(x)\}$ passing through the origin and that $v$ contains $x$ as real analytic parameters. Then $C$ is a real analytic hypersurface on that neighborhood.

We believe that in this lemma distribution can be replaced by hyperfunctions hence in Theorem $L$ the assumption of the weakness of the 
singularity is superfluous.

Conversely we have

Theorem N. Given an $r_{s}{ }^{0}(p)$-dimensional real analytic submanifold $C \subset S$ timelike with respect to $p$, we can construct a real analytic solution $u$ of $p(x, D) u=0$ which has $C$ just as the singularity and unremovable as a hyperfunction solution.

In fact it suffices to find a solution $u$ of $p(x, D) u=\delta\left(x_{1}\right) \delta_{c}\left(x^{\prime}\right)$ real analytic outside $C$, where $\delta_{C}$ denotes the $(n-1)$-dimensional standard measure supported by $C$. This is possible because $p$ is elliptic at the conormal elements of $\boldsymbol{C} \subset \boldsymbol{R}^{n}$.

The gap between $r_{S}(p)$ and $r_{S}{ }^{0}(p)$ or between Theorem $\mathrm{L}$ and Theorem $N$ has also to do with the lack of the theory of the irreducible decomposition of $p$.

In the above we treated the most generic case. We expect that in more degenerate cases the shape of the singularity may be more restricted and interesting relation with analytic geometry may occur. For those singularity not contained in an analytic hypersurface we have no tool at present. We only remark that the example of the ultrahyperbolic operator $p=D_{1}{ }^{2}+\cdots+D_{k}{ }^{2}-D_{k+1}^{2}-\cdots D_{n}{ }^{2}$ shows that the minimal dimension $r_{S}(p)=r_{S}{ }^{0}(p)=n-k$ depends on the choice of $S$. It is very probable that $r(p)=\min \{k, n-k\}$.

\section{References}

[1] Bony, J. M. and Schapira, P., Solutions hyperfonctions du problème de Cauchy, Lecture Notes in Math. 287 (1973), Springer, pp. 82-98.

[2] Grušin, V. V., On solutions with isolated singularities for partial differential equations with constant coefficients, Trudy Moskov. Mat. Ob̌sč. 15 (1966), 262-278 (in Russian).

[3] John. F., Plane Waves and Spherical Means, Interscience, 1955.

[4] Kaneko, A., On continuation of regular solutions of partial differential equations to compact convex sets, J. Fac. Sci. Univ. Tokyo Sec. IA 17 (1970), 567-580.

[5] Kaneko, A., Fundamental Principle and extension of solutions of linear partial differential equations with constant coefficients, Lecture Notes in Math. 287 (1973), Springer, pp. 122-134.

[6] Kaneko, A., On continuation of regular solutions of partial differential equations with constant coefficients, J. Math. Soc. Japan 26 (1974), 92-123.

[7] Kaneko, A., On linear exceptional sets of solutions of linear partial differential 
equations with constant coeffients, Publ. RIMS Kyoto Univ. 11 (1975), 441-460.

[8] Kaneko, A., Note on continuation of real analytic solutions of partial differential equations with constant coefficients, Proc. Japan Acad. 51 (1975), 262-264.

[9] Kaneko, A., On continuation of regular solutions of linear partial differential equations with real analytic coefficients, Proc. Japan Acad. 51 (1975), Suppl. 798-801.

[10] Kaneko, A., Singular spectrum of boundary values of solutions of partial differential equations with real analytic coefficients, Sci. Pap. Coll. Gen. Educ. Univ. Tokyo 25 (1975), 59-68.

[11] Kaneko, A., Analyticity of minimal dimensional singularity of real analytic solutions, Sci. Pap. Coll. Gen. Educ. Univ. Tokyo 26 (1976), 1-5.

[12] Komatsu, H. and T. Kawai, Boundary values of hyperfunction solutions of linear partial differential equations, Publ. RIMS Kyoto Univ. 7 (1971), 95-104.

[13] Sato, M., T. Kawai and M. Kashiwara, Microfuctions and pseudo-differential equations, Lecture Notes in Math. 287, (1973), Springer, pp. 265-529. 
